\section{Informe sobre la situación de los programas de paludismo en las Américas, $1998^{1}$}

1 Elaborado a partir del "Informe de la situación de los programas de malaria en las Américas" que se presentó ante el 41. ${ }^{\text {er }}$ Consejo Directivo de la OPS, San Juan, Puerto Rico, 27 de septiembre a 1 de octubre de 1999. (Documento CD41/INF/1[Esp.]).
En 1998, 308 de los 803 millones de habitantes de las Américas (38,4\%) vivían en zonas con condiciones ecológicas propicias para la transmisión de la malaria o paludismo. De los 37 países y territorios miembros de la OPS, 21 tienen zonas con transmisión activa de la enfermedad y todos ellos están reorientando sus programas de control de acuerdo con las directrices de la Estrategia Global para el Control del Paludismo (EGCP), adoptada en Amsterdam en 1992, que requiere un cambio de énfasis desde la lucha antivectorial hacia el manejo de casos y se basa en cuatro principios: 1) diagnóstico temprano y tratamiento inmediato; 2) aplicación de medidas de protección y prevención dirigidas al individuo, a la familia y a la comunidad, incluida la lucha antivectorial; 3) desarrollo de la capacidad de prevención y contención de epidemias, y 4) fortalecimiento de la capacidad local de investigación básica y aplicada para promover la evaluación sistemática de la situación y, en particular, los factores ecológicos, sociales y económicos determinantes de la enfermedad.

En consecuencia, los países de las Américas han redefinido sus áreas con riesgo de paludismo en función del nivel de exposición al riesgo de transmisión, que se ha definido como "bajo", "mediano" o "alto" y es el resultado de la interacción de factores relacionados con los desplazamientos humanos, la estabilidad social y la adopción de actitudes y comportamientos individuales y colectivos que previenen el contacto con los vectores. El producto de la interrelación de estos factores es la intensidad de transmisión del paludismo, que puede ser modificada por el acceso a servicios adecuados de diagnóstico y tratamiento, y que se refleja aproximadamente en el índice parasitario anual (IPA, expresado como número de casos por 1000 habitantes), la variable básica utilizada para estratificar epidemiológicamente las zonas de paludismo endémico. Como índice de morbilidad, para facilitar las comparaciones con otros índices de morbilidad, se ha utilizado la "detección de casos", que se expresa como número de casos por 100000 habitantes y constituye una variante del IPA, de uso más generalizado en los programas de control del paludismo.

La "detección de casos" aumentó entre 1997 y 1998 de 135,5 a 160,51/100 000 en la población total 
de las Américas, y de 350,8 a 418,31/100 000 en la población residente en zonas ecológicamente propicias para la transmisión de la enfermedad. En los 21 países de la Región con paludismo endémico, al comparar el IVA y el IFA (índices similares al IPA, pero referidos por separado a los casos causados por Plasmodium vivax y por P. falciparum) con el índice anual de exámenes de sangre se observa una tendencia al aumento de la proporcion de infecciones por $P$. vivax notificadas a medida que aumenta el número de frotis sanguíneos examinados, hecho que podría reflejar una cobertura terapéutica incompleta del reservorio de $P$. vivax. En cambio, no ocurre lo mismo con P. falciparum, cuya transmisión se controla más eficazmente con el tratamiento inmediato tras el diagnóstico de laboratorio.

De los 460 millones de habitantes de los 21 países con transmisión activa del paludismo, 219 millones $(47,6 \%)$ viven en zonas expuestas a algún riesgo de transmisión y, de estos 219 millones, 131 están expuestos a un riesgo bajo o extremadamente bajo. En estas regiones, en 1998 se detectaron 53778 casos de paludismo, lo cual supone un aumento de 45,6\% en comparación con los 36934 casos registrados en 1997. Este aumento se debió a la epidemia que tuvo lugar a consecuencia del fenómeno meteorológico "El Niño" en Colombia, Ecuador y Perú. El 19\% (88 millones) de la población de estos 21 países vive en zonas con riesgo moderado a elevado de transmisión del paludismo. El incremento de la población de alto riesgo (de 31 a 39 millones entre 1997 y 1998) se debió fundamentalmente a la reestructuración de las áreas de riesgo en México. En la poblaciones de la Región con riesgo moderado a elevado de transmisión, el IPA oscila entre 0,42/1 000 en México y 310,2/1 000 en Suriname. El mayor número de casos se notificó en la subregión andina $(44,4 \%)$, y, por países, en Brasil $(36,6 \%)$. Sin embargo, el mayor riesgo de transmisión correspondió a la subregión de Guyana, Guyana Francesa y Suriname (IPA = 101,3/1 000), seguida de algunas zonas de Brasil (IPA = 64,0/1 000).

Recientemente, la estratificación de la situación epidemiológica del paludismo en las Américas ha permitido integrar la detección de casos, el diagnóstico y el tratamiento inmediato en los servicios locales de salud. De los 6,5 millones de casos sospechosos de paludismo identificados por los servicios locales de salud $(74 \%)$ y por el sistema de búsqueda activa de casos $(26 \%)$, solo se confirmaron parasitológicamente $11,2 \%$ de los primeros y $3,4 \%$ de los segundos, a pesar del alto costo operativo de este último sistema. La disponibilidad de tratamiento oscila entre 0,77 y 310,2 tratamientos por caso notificado. La mejor disponibilidad de tratamientos de segunda y tercera línea en Bolivia, Brasil, Colombia, Guyana, Perú y Venezuela en comparación con años anteriores puede deberse a la cobertura más amplia de la EGCP.

La caracterización de los factores que perpetúan la transmisión de la enfermedad permite identificar posibles medidas de control, pero para garantizar su aplicación y sostenibilidad es necesario seguir mejorando su selección y orientación, además de movilizar, orientar y respaldar la coordinación intersectorial. La lucha antivectorial, limitada casi exclusivamente a la fumigación intradomiciliaria de insecticidas de acción residual, sigue siendo la principal herramienta utilizada por los países para prevenir la transmisión. En este aspecto, se observa una clara tendencia al abandono de los insecticidas organoclorados y organofosforados en favor de los piretroides sintéticos. Los fondos utilizados por los programas de control fueron muy variables en los últimos cinco años, pero el gasto por persona en las zonas palúdicas sigue disminuyendo. En los 16 países de la Región que en 1998 notificaron a la Organización Panamericana de la Salud (OPS) su presupuesto para el control del paludismo, el gasto fue de 0,40 dólares estadounidenses/persona, lo cual representa una disminución de $50 \%$ con respecto a los US\$ 0,80 de 1995 y de $35 \%$ con respecto a los US\$ 0,65 de 1996.

\section{PRINCIPALES CAMBIOS EPIDEMIOLÓGICOS}

La distribución epidemiológica del paludismo en las Américas ha sufrido cambios, entre los que destacan la disminución del paludismo por $P$. falciparum en la Amazonia brasileña, debida sobre todo a la mejoría del diagnóstico precoz y tratamiento inmediato de los casos, a la reducción de la actividad minera no controlada y a la disminución de los desplazamientos humanos. En cambio, en las zonas boscosas de la subregión andina, principalmente en la cuenca amazónica, y en la costa del Pacífico de Colombia, Ecuador y Perú ha habido un aumento de la prevalencia de paludismo por $P$. falciparum, fenómeno que se vio agravado por las intensas lluvias ocasionadas por "El Niño" en 1997 y 1998. La puesta en marcha de la EGCP ha ocasionado una significativa disminución de la tasa de mortalidad específica por paludismo. La tasa bruta de mortalidad por $P$. falciparum disminuyó de 8,3 a 3,0/100 000 habitantes expuestos entre 1994 y 1998, lo cual representa una reducción de $64 \%$, fenómeno asociado al aumento constante de la cobertura de los tratamientos de segunda y tercera línea. 


\section{RESISTENCIA DE P. FALCIPARUM A LOS ANTIPALÚDICOS}

La resistencia a la cloroquina está muy difundida en Sudamérica y el número notificado de fracasos del tratamiento ha aumentado hasta acercarse a $20 \%$ en algunas zonas de la Amazonia peruana. La resistencia a la sulfadoxina/pirimetamina también está muy difundida. En Colombia y las Guyanas se están utilizando cada vez más la quinina, y las tetraciclinas como fármacos de primera línea, y en la Amazonia brasileña se ha empezado a utilizar la mefloquina como tratamiento de primera línea frente a P. falciparum. Los derivados de la artemisina se siguen reservando para los casos graves y complicados. Informes recientes de fracasos aislados del tratamiento con cloroquina en casos de paludismo por $P$. vivax todavía no han sido confirmados por estudios epidemiológicos.

\section{PRINCIPALES PROBLEMAS Y LIMITACIONES}

Las principales barreras al éxito del control del paludismo son la idea imperante en el campo de la salud pública de que la enfermedad se controla mediante la fumigación con insecticidas, la reducción drástica de los presupuestos centrales a consecuencia del proceso de descentralización de los servicios de salud y la inexistencia de un control eficaz de los vectores o de una medida eficaz de interceptación de los mismos.

\section{REDUCCIÓN DE LOS PROBLEMAS Y LIMITACIONES}

La respuesta a estos problemas ha tratado de orientarse siempre hacia la selección del sistema de prestación de asistencia sanitaria más sostenible, capaz de funcionar pese a la descentralización y a los constantes cambios de prioridades creados por las sucesivas "epidemias" y otras situaciones de emergencia. Los países de la Región han empezado a reforzar la red de colaboradores voluntarios, que había perdido apoyo con el transcurso de los años. Por otro lado, la reforma del sector de la salud no afectó a la distribución privada de medicamentos antipalúdicos, que sigue funcionando al margen de los principios básicos de la EGCP, y requerirá una concentración de esfuerzos para hacerla coincidir con las prioridades sanitarias nacionales. La reglamentación legislativa y técnica exige cada vez más cooperación técnica.

\section{LA INICIATIVA “HACER RETROCEDER EL PALUDISMO" (RBM) EN LAS AMÉRICAS}

Esta iniciativa, introducida en 1998 por la Organización Mundial de la Salud (OMS), complementará las actividades de la EGCP. Su objetivo general consiste en reducir significativamente la carga mundial de paludismo mediante intervenciones adaptadas a las necesidades locales, y para alcanzarlo se han definido cinco componentes cruciales: intervenciones estructuradas, integración de recursos, política farmacéutica antipalúdica definida, sistema operativo de derivación de casos para diagnóstico y tratamiento y creación de redes de recursos. En las Américas se considera necesario también un sexto elemento: el control de la transmisión del paludismo.

\section{Intervenciones estructuradas}

Los 21 países de la Región con paludismo endémico han organizado programas de lucha contra esta enfermedad con niveles diferenciados de integración dentro de sus servicios generales de salud. El nivel de descentralización de responsabilidades en cuanto a planificación y formulación de presupuestos varía de un país a otro. En general, son los municipios los que se encargan de llevar a cabo las actividades de salud pública a nivel local y algunos de ellos (muy pocos) ya tienen control sobre los recursos financieros. La descentralización de responsabilidades no se ha visto acompañada necesariamente por una descentralización de las capacidades técnicas, lo cual provocó por lo general una pérdida de personal adiestrado. Es muy necesario establecer capacidades técnicas a este nivel de ejecución de las actividades de control, que, además, deberían incluir la amplia participación de las autoridades locales para planificar, formular presupuestos y compartir esas capacidades dentro de los servicios de salud locales.

\section{Integración de recursos}

El plan de Agentes Comunitarios de Salud, que incluye voluntarios en algunos casos y trabajadores remunerados en otros, ha sido objeto de un fomento intenso en la Región y, allí donde se ha establecido, ha resultado ser muy útil para ampliar la cobertura de los servicios generales de salud. Los grupos más afectados por el paludismo son los de las zonas periféricas dotadas de una infraestructura de atención sanitaria limitada. Hay que seguir ela- 
borando materiales didácticos que permitan fomentar comportamientos favorecedores de la salud. Se prestará especial atención a las asociaciones dirigidas a mejorar la detección y el tratamiento de la enfermedad. Las alianzas entre programas de lucha contra el paludismo y programas comunitarios como los de promoción de la salud en las comunidades y la Atención Integrada a las Enfermedades Prevalentes de la Infancia pueden mejorar el acceso de los hogares. La educación sanitaria y la promoción de la salud, dirigidas hacia el reconocimiento de lo importante que es buscar tratamiento temprano para las fiebres, fomentarán la demanda de un diagnóstico y tratamiento accesibles de la enfermedad, en conformidad con la EGCP.

\section{Políticas sobre medicamentos antipalúdicos}

La vigilancia de la eficacia de los medicamentos antipalúdicos en los diferentes marcos geográficos y sociales de la Región y la definición de regímenes terapéuticos específicos en función de las situaciones locales constituyen la base para velar por la disponibilidad y el control de calidad de los medicamentos antipalúdicos. Se han elaborado protocolos sobre la eficacia de los medicamentos y en ocho centros de seis países de la Región (Brasil, Colombia, Guyana, Perú, Suriname y Venezuela) se están realizando pruebas que se seguirán promoviendo para garantizar una vigilancia y evaluación permanentes de la eficacia de los medicamentos y la formulación constante de regímenes alternativos, en función de la sensibilidad local.

\section{Sistema de derivación de casos}

Se han fortalecido las posibilidades de diagnóstico y tratamiento de los servicios generales de salud en Brasil, Colombia y Venezuela, y en estos momentos se está reforzando en Perú, Suriname y Bolivia la capacidad de manejar casos graves y complicados con el objetivo de ampliar las posibilidades de acceso de la población tanto a la vigilancia y diagnóstico satisfactorios de los fracasos del tratamiento como al tratamiento de los casos graves y complicados. La OPS/OMS ha elaborado y divulgado materiales para uso clínico, cuyo empleo permitirá seguir reduciendo la mortalidad. El control periférico mejorado del paludismo no complicado y la disminución de la incidencia de la enfermedad mediante el control de su transmisión seguirán reduciendo la aparición de casos graves y complicados, para lo cual también será necesaria una actualización constante del personal sanitario y del sistema de derivación de casos, adaptándolo a las distintas situaciones epidemiológicas.

\section{Redes de recursos}

En el marco de la iniciativa RBM se establecerán redes de recursos para brindar apoyo directo a las actividades de control y tratar las cuestiones de importancia básica para las políticas de lucha antipalúdica. Se busca fomentar así la colaboración dentro de los países y entre los mismos, la divulgación de las enseñanzas aprendidas y la utilización óptima de los conocimientos especializados y las experiencias regionales. De las redes de recursos establecidas por la iniciativa, las de importancia particular para la Región son las de prevención y control de epidemias, calidad y suministro de medicamentos antipalúdicos a nivel local y vigilancia de la resistencia a los medicamentos antipalúdicos y a los insecticidas. Además se deberá establecer una red para la validación y el mejoramiento de otros métodos posibles de control selectivo de la transmisión. Concretamente, la red para la prevención y el control de epidemias funcionará en estrecha colaboración con los programas de preparación y mitigación de desastres a nivel internacional, nacional y local. También es necesario un mayor financiamiento para poder continuar las actividades en curso e iniciar otras.

\section{Control de la transmisión del paludismo}

Mientras persisten en sus esfuerzos para reducir la mortalidad por paludismo, los países de la Región están tratando de alcanzar el objetivo adicional de reducir la incidencia de la enfermedad mediante el control de su transmisión, por ejemplo mediante el uso de materiales impregnados de insecticidas. No obstante, las actividades de control de la transmisión siguen constituyendo un gran reto para los servicios de salud descentralizados de la Región. La fumigación intradomiciliaria de insecticidas fue eficaz en grandes zonas de la Región, especialmente las de desarrollo socioeconómico continuo, pero no tuvo tanto éxito en algunas zonas endémicas, principalmente con situación sociodemográfica y política inestable, donde hoy se siguen buscando activamente otros métodos de control de la transmisión.

Plan de Acciones Intensivas y Simultáneas (PAIS). En las zonas endémicas donde la población en riesgo es relativamente estable y en las zonas de nuevos asentamientos rurales y urbanos 
periféricos, la transmisión puede reducirse utilizando medidas antiparasitarias simultáneas en los reservorios humanos y vectoriales. Esta técnica, perfeccionada en México, exige una organización con una infraestructura nacional para poder ejecutar el plan PAIS de manera eficiente y sistemática en todas las comunidades del país con paludismo endémico. Los costos pueden ser considerablemente elevados para algunos países, pero la mayoría de ellos han logrado financiarlos. El cálculo realizado por México es de US\$ 40 millones anuales durante 6 años, con un costo unitario por persona en riesgo de contraer paludismo de US\$1,00.

Control selectivo de vectores. En áreas de expansión de la "frontera económica" en regiones boscosas sometidas a la presión de los asentamientos humanos y con una baja explotación tecnológica de los recursos naturales, la fumigación intradomiciliaria de insecticidas ha tenido un efecto muy limitado. Un comité regional de expertos en control selectivo de vectores propuso otros métodos de control para esas situaciones, entre los que cabe señalar la reducción de fuentes mediante el control de los criaderos; la promoción de medidas de protección personal, familiar y comunitaria; el control biológico de vectores, y la fumigación de insecticidas en espacios abiertos. La utilización de técnicas de estratificación epidemiológica y entomológica permite asignar prioridades y seleccionar la combinación de métodos más adecuados para cada foco de transmisión del paludismo. La OPS/OMS preparó manuales de capacitación e instrucciones para el personal de los servicios locales de salud que actualmente están siendo puestos a prueba y validados sobre el terreno.

\section{SYNOPSIS}

\section{Report on the situation of malaria programs in the Americas (1998)}

This report analyzes the current situation of malaria programs in the Americas from the perspective of the Global Malaria Control Strategy, which was adopted in Amsterdam in 1992. After describing the current situation with the disease in the Americas, the report analyzes the principal epidemiological changes and the resistance to antimalarials, as well as the major problems and limitations of the disease control programs, and possible solutions. The report also discusses the activities carried out within the context of the Roll Back Malaria (RBM) initiative, which was adopted in 1998 by the World Health Organization. The fundamental components of that RBM effort are structured interventions, the integration of resources, an antimalarials policy, a strong referral system for diagnosis and treatment, resource networks, and programs to control malaria transmission. 